\title{
Green Nanocolloids Control Multi Drug Resistant Pathogenic Bacteria
}

\author{
S. Ranjani ${ }^{1}$ - M. Shariq Ahmed ${ }^{1} \cdot$ K. Ruckmani ${ }^{2}$ - S. Hemalatha ${ }^{1}$
}

Received: 9 July 2019/Published online: 16 October 2019

(C) Springer Science+Business Media, LLC, part of Springer Nature 2019

\begin{abstract}
Cymbopogon citratus is grown globally for therapeutic application is utilized to synthesize colloidal silver nanoparticles (CcAgNps). CcAgNps are characterized by biophysical methods and are tested against CTXM-15 expressing pathogenic Escherichia coli strains. MIC, MBC and biofilm assays are performed at different concentrations of nanoparticles. The expression of CTXM-15 gene responsible for antibiotic resistance is analyzed by polymerase chain reaction to identify the role of CTXM-15 in pathogenesis. CcAgNps show better antibacterial and anti-biofilm activity when compared to antibiotics. This study suggests that CcAgNps are the potential antibiotic resistance breakers to control pathogenic E. coli and can be utilized as alternatives to antibiotics.
\end{abstract}

Keywords Cymbopogon citratus $\cdot$ Antibiotic resistance $\cdot$ CTXM-15

\section{Introduction}

Plants are the potential resources of medicinal compounds. Plants and its compounds have been used from the ancient days as a traditional medicine in the treatment of various ailments [1]. Lemon grass (Cymbopogon citratus) has been utilized for therapeutic applications globally [2]. Lemon Grass is an aromatic grass contains aromatic essential oil called as Cochin oil. Aromatic or essential oils are highly concentrated secondary metabolites of diverse functions in plant system which comprises organic compounds including terpenoids, benzenoids, organic sulphur and nitrogenuous compounds which are useful in treating various health issues. C. citratus leaf extract, essential oil and its constituents namely citral, geraniol, and geranyl acetate have been reported to possess several bioactivities viz., antimicrobial, antiparasitic, nematicidal, antifungal, anti-inflammatory, anticancer, antioxidant, allelopathic, anthelmintic, and insect/mosquito repellent [3-5].

S. Hemalatha

hemalatha.sls@crescent.education

1 School of Life Sciences, B.S.Abdur Rahman Crescent Institute of Science and Technology, Vandalur, Chennai 600048, India

2 Department of Pharmaceutical Technology, University College of Engineering, Anna University BIT Campus, Tiruchirappalli, Tamilnadu 620024, India
Green nanotechnology utilizes nanotechnology for the development of products from plant and plant extracts, which are rapid, ecofriendly, commercially viable and safer to all living beings on the earth $[6,7]$. Nanoparticles are widely synthesized by chemical, electrochemical and green synthesis by bioreduction methods [8]. The green biogenesis of nanoparticles from metals has received greater attention due its unusual physical, chemical, optical and biological properties and widely applied in the diverse areas of health care, agriculture, medicine, food etc. [9]. Silver in nano size is more reactive than metallic silver because of larger surface to volume ratio [10]. This characteristic feature makes the nanoparticles to enter the cell membrane, followed by accumulation leading to cell deaths [11]. There are numerous reports on silver nanoparticles, as a potential antimicrobial agent against bacteria, viruses, fungi and other organisms [12, 13].

The main objective of the present study is to exploit and combine the therapeutic application of noble silver metal and $C$. citratus leaf extract to synthesize silver nanoparticle from $C$. citratus leaf extract using rapid biogenesis method. Biophysically characterized colloidal CcAgNps were used to test the efficacy on CTXM-15 positive $E$. coli and its CTXM-15 gene expression studies. Because "The CTX-M pandemic" is E. coli producing CTXM enzyme emerged globally due to onset of UTI's [14]. The most widely distributed is CTXM-15 enzyme which was detected in 2001. CTXM is mostly responsible for hospital based acquired 
infections. CTX-M beta-lactamases hydrolyse ceftriaxone and cefotaxime and more specifically ceftazidime [15]. CTXM-15 type Extended spectrum beta lactamases (ESBLs) were detected in K. pneumoniae, E. coli and Enterobacter aerogenes from India and Japan. CTXM-15 type ESBLs are more prevalent in southern part of India [16, 17]. Hence, CTXM-15 encoding clinical strains of E. coli was used for the present study to exploit the antibacterial effect of $C$. citratus based silver nanoparticles.

\section{Materials and Methods}

The E. coli strains such as ATCC (25922) and clinical isolates with and without CTXM-15 E. coli MDR strains were isolated from UTI samples (urinary tract infection) obtained from Tagore Medical College and Hospital, Chennai after proper ethical approval from BSACIST ethical committee (Ref. no. BSAU: REG-OFF: 2016/02SLS).

\section{Preparation of Cymbopogon citratus Leaf Extract}

The dried Cymbopogon citratus leaves extract was prepared through following steps. $5 \mathrm{~g}$ of dried leaves was ground in a mixer into fine powder. $5 \mathrm{~g}$ of leaf powder was dissolved in $100 \mathrm{~mL}$ of sterile distilled water $(5 \% \mathrm{w} / \mathrm{v})$. The suspension was kept at $37^{\circ} \mathrm{C}$ for overnight at 100 RPM in the incubator shaker (Scigenics biotech pvt. Ltd). The solution was concentrated, by boiling it for $30 \mathrm{~min}$ at $80{ }^{\circ} \mathrm{C}$. The extract was allowed to cool and finally filtered through Whatman No: 1 filter paper $[12,13]$. The filtrate was stored in refrigerator at $4{ }^{\circ} \mathrm{C}$ for future use.

\section{Biosynthesis and Physico-Chemical Characterization of Cymbopogan citratus Silver Nanoparticles Using Microwave Irradiation Method}

The Cymbopogan citratus (Lemon grass) aqueous extract of $100 \mathrm{~mL}$ was mixed with equal volume of $1 \mathrm{mM}$ Silver nitrate solution. Then the flask was subjected to microwave irradiation for $2 \mathrm{~min}$. The colour change was observed for visual examination of nanoparticles synthesis. After confirming the synthesis of CcAgNps, then the solution was subjected to centrifugation at $15,000 \mathrm{rpm}$ for $20 \mathrm{~min}$ at $4{ }^{\circ} \mathrm{C}$. The pellet was washed with autoclaved distilled water twice and resuspended in 30\% DMSO. Then CcAgNps was subjected to sonication for $10 \mathrm{~min}$. The synthesis of colloidal Cymbopogan citratus silver nanoparticles through the bioreduction method was confirmed by obtaining spectrum of UV-vis spectroscopy, between 300 and $700 \mathrm{~nm}$. FTIR was also carried out for crude extract of the Cymbopogan citratus and $\mathrm{AgNps}$ to identify the presence of potential biomolecules. (Perkin Elmer Spectrum100) in the wavelength range of $4000-400 / \mathrm{cm}$ [18]. CcAgNps was subjected to Dynamic Light Scattering analysis for determination of size of the nanoparticles (Malvern Instruments Ltd) and the stability was checked by observing the Zeta Potential (Malvern Instruments Ltd) [19]. The morphology and shape of the AgNps were examined using field emission electron microscopy (SIGMA HV-Carl Zeiss with Bruker Quantax 200-Z10 EDS Detector) and EDAX analysis [19].

\section{Assessment of MIC, MBC and Biofilm Formation}

The MIC of CcAgNps was carried out in a 96 well microtitre plate. $100 \mu \mathrm{L}$ of Lysogeny Broth (LB) was treated with $100 \mu \mathrm{L}$ of the $\mathrm{CcAgNp}(1 \mathrm{mg} / \mathrm{mL})$ by serial dilution and finally the bacterial strain was inoculated. Bacterial culture without $\mathrm{CcAgNp}$ treatment was kept as positive control. Lysogeny Broth and $\mathrm{CcAgNp}$ was used as negative control. 96 well microtitre plates were incubated in Incubator shaker (Scigenics biotech pvt. Ltd) for overnight at $100 \mathrm{RPM}$, maintained at $37{ }^{\circ} \mathrm{C}$. The visual turbidity was observed before and after incubation with all the bacterial strains, which confirmed the MIC value of CcAgNps. From the recorded results of CcAgNp-MIC, the wells which showed no turbid growth were chosen. Each selected well $(2.0 \mu \mathrm{L})$ were inoculated in LB agar plates by drop plate method for checking the MBC [17]. Then the plates were incubated for $24 \mathrm{~h}$ at $37^{\circ} \mathrm{C}$. MBC was observed for presence and absence of bacterial growth [16]. Biofilm assay was performed in a 96 well microtiter plate. Here in $100 \mu \mathrm{L}$ of LB broth and $100 \mu \mathrm{L}$ of the CcAgNp $(1 \mathrm{mg} / \mathrm{mL})$ was added in each well and serially diluted and finally the cultures were inoculated in all wells and kept in incubator shaker at $37{ }^{\circ} \mathrm{C}$ for up to $48 \mathrm{~h}$. After incubation the used medium from the plate was removed completely. Then the plate was washed with distilled water and blotted it dry using filter paper. Crystal violet $(0.1 \%)$ solution was added into each well and left it for $15 \mathrm{~min}$ for incubation, and then the staining solution was discarded by inverting the plates and washed with water and left it to dry by inverting the plates on a paper overnight/until its dry and free of liquid crystal violet. Followed by the addition of $30 \%$ Glacial acetic acid to solubilize the crystal violet in each well and left for 10 min in shaker for completely dissolving the crystal violet and the absorbance measured at $595 \mathrm{~nm}$ using Enspire multi label plate reader with Alpha Tech (Perkin Elmer, USA) [16]. 


\section{Isolation of DNA and CTXM-15 Gene Expression Studies Using Polymerase Chain Reaction (PCR)}

ATCC (25922) CTXM-15 Negative, CTXM-15 positive strains of E. coli were inoculated in LB broth, served as a control. The same set of strains, which was treated with $10 \mu \mathrm{g} / \mathrm{mL}$ of CcAgNps, is considered as treated. Both the control and treated strains were incubated at $37^{\circ} \mathrm{C}$ overnight. From the inoculated strains $500 \mu \mathrm{L}$ of broth was taken in $1.5 \mathrm{~mL}$ microcentrifuge tube, for the control and treated tubes. The DNA isolation was carried out, using heat lysis method with some modification [20]. The culture was spin down at 12,000 rpm for $5 \mathrm{~min}$. The supernatant was discarded from the tube and the pellet was washed with autoclaved distilled water. Finally, $500 \mu \mathrm{L}$ of autoclaved distilled water was added to the pellet and mixed well. Then the tubes were placed in a thermostat at $95{ }^{\circ} \mathrm{C}$ for $10 \mathrm{~min}$ and cooled. The tubes were centrifuged at $3000 \mathrm{rpm}$ for $5 \mathrm{~min}$ [21]. The supernatant was transferred to another tube and checked in $0.8 \%$ Agarose gel electrophoresis. This DNA samples from all isolates were utilized for the amplification of CTX-M-15 gene. The amplification reaction was carried out (Mastercycler nexus gradient, Eppendorf, USA) in $10 \mu \mathrm{L}$ reaction mixtures comprises of $5 \mu \mathrm{L}$ of $2 \times$ Redeye Master Mix (Amplicon III), $1 \mu \mathrm{L}$ of $1 \mathrm{M}$ CTX-M-15 forward (CACGTCAATGGGACGATGT) and $1 \mu \mathrm{L}$ of $1 \mathrm{M}$ CTX-M-15 reverse (GAAAGGCAATACCACCGGT) primer, $2.0 \mu \mathrm{L}$ of Milliq water and $1.0 \mu \mathrm{L}$ of template DNA. The amplification reaction was performed with the following conditions: initial denaturation at $94{ }^{\circ} \mathrm{C}$ for $5 \mathrm{~min}$, accompanied by 35 cycles of denaturation at $94{ }^{\circ} \mathrm{C}$ for the $30 \mathrm{~s}$, annealing at $55^{\circ} \mathrm{C}$ for $45 \mathrm{~s}$ and extension at $72{ }^{\circ} \mathrm{C}$ for $45 \mathrm{~s}$ and final extension at $72{ }^{\circ} \mathrm{C}$ for $5 \mathrm{~min}[15,21]$. The PCR product was checked by electrophoresis in $1.5 \% \mathrm{w} / \mathrm{v}$ agarose gel at $60 \mathrm{~V}$ for $60 \mathrm{~min}$ using $1 \times$ Tris-acetateEDTA buffer (1× TAE buffer), $\mathrm{pH}-7.6,20 \mathrm{mM}$ acetic acid, $1 \mathrm{mM}$ EDTA), using ethidium bromide as intercalating agent and $1 \mathrm{~kb}$ DNA ladder (Gene Direx) as a marker. The gel was imaged under ChemiDoc MP System (Bio-Rad, USA) after $1 \mathrm{~h}$ of electrophoresis (Biorad, USA).

\section{Results and Discussion}

CcAgNps was prepared from the aqueous extract of Cymbopogon citratus extract using $1 \mathrm{mM}$ silver nitrate solution. It was reported that the dried leaves of Cymbopogon citratus contains more phenol and flavonoids than fresh one [2]. These phytochemical components could be incorporated during nanoparticles synthesis. For the synthesis of silver nanoparticles $100 \mathrm{~mL}$ of Cymbopogon citratus leaves extract and $100 \mathrm{~mL}$ of $1 \mathrm{mM}$ silver nitrate solution was mixed together. No color change was observed. Then the solution was subjected to microwave irradiation for $2 \mathrm{~min}$. There was an immediate color change from light greenish brown to dark brown color. The phenomenon of surface excitation of plasmon resonance is due to colour change of the aqueous solution. There are articles reporting that the synthesis of silver nanoparticles was fastened by the temperature [7]. Extract of C. citratus contains saponins, tannins, anthroquinines, alkaloids, steroids, phenols and flavonoids. The phytoconstituents of $C$. citratus include citral, z-citral, borneol, estragole, methyleugenol, geranyl acetate, geraniol, beta-myrcene, limonene, piperitone, citronellal, carene-2, alpha-terpineole, pinene, farnesol, proximadiol, and cymbodiacetal. These bioactive phytoconstituents act as capping agent to protect them from aggregation in aqueous extract during silver nanoparticles synthesis [22].

The size and stability of CcAgNps in solution was obtained from UV-vis spectroscopy. After $2 \mathrm{~min}$ of microwave irradiation the UV-vis spectrum of CcAgNps was taken (Fig. 1a). The absorption spectra of CcAgNPs were scanned between 200 and $700 \mathrm{~nm}$ and a peak at $350 \mathrm{~nm}$ was observed which confirmed the biogenesis of silver nanoparticles capped with aqueous extract of $C$. citratus. The biogenesis of CcAgNps was confirmed by observing the peak within the range of SPR for CcAgNPs. FT-IR was used to characterize the biomolecules responsible for reducing and capping of the biosynthesized CcAgNps. The FT-IR absorption bands of the crude extract and biosynthesized $\mathrm{CcAgNps}$ between the range of 400-4000 $\mathrm{cm}^{-1}$ (Fig. 1b, c). Biosynthesized CcAgNps showed different stretches with varying functional groups including alkanes, alkynes, nitriles and carboxyl groups. The Zeta potential analysis showed the aggregation of nanoparticles through its surface charge and mobility. Our results showed that prepared $\mathrm{CcAgNps}$ have enough negative charge and mobility to inhibit aggregation of nanoparticles. Zeta potential value was found to be negative -25.7 for CcAgNps (Fig. 1d). Negative charge revealed that repulsion of charged particles enhanced the stability of nanoparticles by reducing the aggregation [23]. This makes the nanoparticles to behave as separate entities in the suspension of nanoparticles. The range of nanoparticles size was determined using particle size analyzer (Fig. 1e). The particle size was found to be $797.3 \mathrm{~nm}$, which was based on the Brownian motion undergone by the CcAgNps, under the influence of laser light scattering method. FESEM results showed the surface morphology of CcAgNps, which was found as spherical shaped nanoparticles (Fig. 1f). The phytochemical compounds present in the $C$. citrates leaf extract acted as capping and reducing 
A

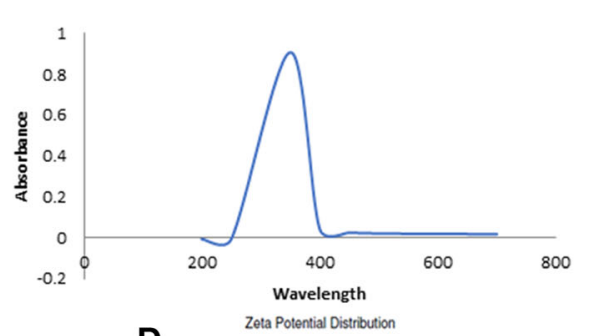

D

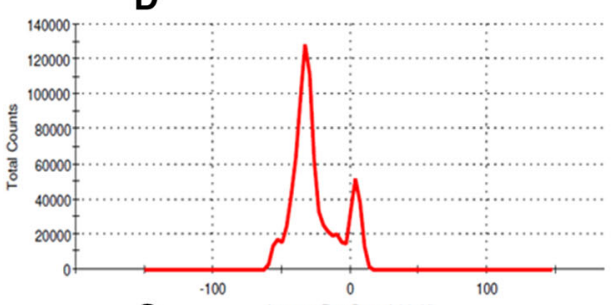

G

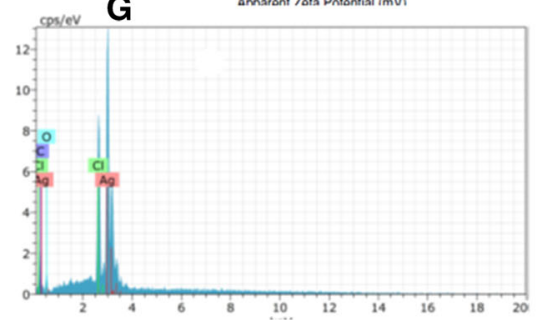

B

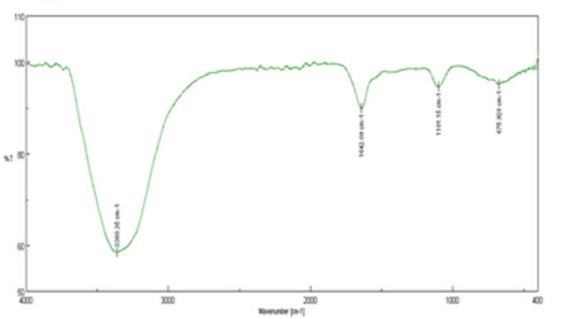

E

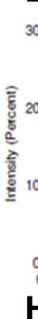

H
C
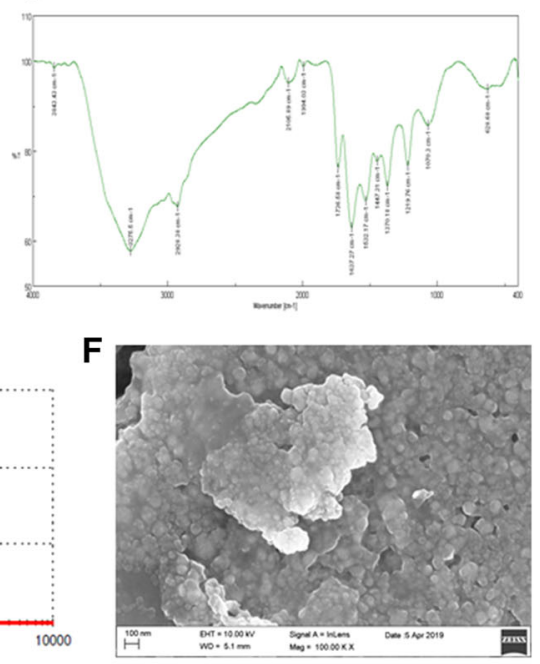

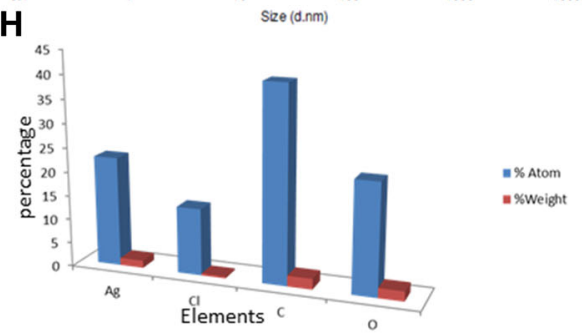

CcAgNps (g). EDAX spectra of CcAgNps (h). Histogram showing the percentage atom and percentage weight of $\mathrm{Ag}$ in $\mathrm{CcAgNps}$

Biophysical characterization of $\mathrm{CcAgNps}(\mathbf{a})$. Uv-Vis absorp tion spectra of CcAgNps (b). FT-IR of crude extract (c). FT-IR of $\mathrm{CcAgNps}(\mathbf{d})$. Zeta potential of CcAgNps (e). Particle size distribution of CcAgNps under Brownian movement (f). FESEM Image of

agent during the synthesis of silver nanoparticles. The results from the energy dispersive spectrum (Fig. 1g) of the CcAgNps, confirmed the presence of silver as the predominant element, by showing the very strong signal peak at $3 \mathrm{keV}$, which indicates the synthesis of CcAgNps. Since the surface plasmon resonance of metallic silver will show strong signal peak at $3 \mathrm{keV}$. EDAX results depicted the information of percentage weight and percentage atom of CcAgNps. This confirmed that the percentage weight and percentage atom of $\mathrm{Ag}$ is high, which supports the antibacterial mechanism of CcAgNps (Fig. 1h).

In this present study, MIC was carried out to determine the lowest concentration of CcAgNps that completely inhibits the visible growth of $E$. coli. MIC was evaluated for the CcAgNps against 6 different strains of $E$. coli including-ATCC (25922), one strain of CTXM-15 Negative and 4 different strains of CTXM-15 positive. There were distinct differences among all the strains. CTX-M-15positive E. coli are resistant to all oral $\beta$-lactam antibiotics, as well as quinolones and sulfonamides. The results show that, CcAgNps had better antimicrobial activity against all the six strains of $E$. coli however it was concentration dependent (Table 1). Minimum Inhibitory Concentration for E. coli ATCC (25922) E. coli and CTXM-15 negative

strains were found as $3 \mu \mathrm{g} / \mathrm{mL}$ and $6.25 \mu \mathrm{g} / \mathrm{mL}$ respectively, Whereas Minimum Inhibitory Concentration for E. coli CTM15 positive strain were $12.5 \mu \mathrm{g} / \mathrm{mL}$ and $25 \mu \mathrm{g} /$ $\mathrm{mL}$ which was higher than that required for ATCC and CTXM-15 negative strains. The MIC results show that the percentage growth of all the six strains are considerably reduced when compared to the control and ampicillin treated strains (Fig. 2A). The E. coli ATCC (25922) and CTXM-15 negative strains are sensitive to both antibiotics and $\mathrm{CcAgNps}$, which resulted in reduction in growth. In CTXM-15 positive strains, the percentage growth was decreased by $20 \%, 40 \%, 70 \%$, and $90 \%$ respectively when

Table 1 Determination of MIC and MBC of CcAgNps against E. coli

\begin{tabular}{llll}
\hline S. no & E. coli strains & MIC $(\mu \mathrm{g} / \mathrm{mL})$ & MBC $(\mu \mathrm{g} / \mathrm{mL})$ \\
\hline 1 & ATCC strain & 3 & 3 \\
2 & CTXM-15 negative & 6.25 & 12.5 \\
3 & CTXM-15 positive 1 & 12.5 & 50 \\
4 & CTXM-15 positive 2 & 12.5 & 25 \\
5 & CTXM-15 positive 3 & 25 & 50 \\
6 & CTXM-15 positive 4 & 25 & 50 \\
\hline
\end{tabular}


compared to antibiotics treated strains. Similarly, the minimal bactericidal concentration was observed as $3 \mu \mathrm{g} /$ $\mathrm{mL}$ for ATCC (25922) E. coli, $12.5 \mu \mathrm{g} / \mathrm{mL}$ for CTXM- 15 Negative E. coli and maximum $50 \mu \mathrm{g} / \mathrm{mL}$ for CTXM- 15 positive $E$. coli strains. The results suggested that CcAgNps were able to penetrate the bacterial cell wall thereby inhibits the bacterial growth. Since all the tested strains were resistant to antibiotics, they are resistant to ampicillin and no inhibition in growth was observed. Interestingly, the CcAgNps controlled the growth of the CTXM-15 encoding E. coli strains which suggested that the CcAgNps could have interfered with normal growth, metabolism, transcription and translation events which eventually arrested the growth of E. coli. Similar kind of results was observed by previous workers [16].

Different concentrations of the CcAgNps are used to check the biofilm inhibition potential against all the six strains of $E$. coli. CcAgNps inhibited biofilm formation in all the bacterial strains compared to control (Fig. 2B). This elicited a dose dependent activity. The results showed that $100 \mu \mathrm{g} / \mathrm{mL}$ of the CcAgNps was more effective than
$50 \mu \mathrm{g} / \mathrm{mL}$, in inhibiting the biofilm formation, due to the presence of phytoconstituents and polyphenolic bioactive compound, which were capped and reduced by silver. It has been reported that green silver nanoparticles inhibited the biofilm formation by damaging the bacterial membrane structure and by disrupting quorum sensing [14]. CcAgNps acted as potential quorum quenching compounds which were due to the presence of phytochemical compounds of plant extract incorporated during nanoparticle synthesis [24]. Our results indicated that CcAgNps can able to combat the biofilm formation by fivefold when compared to the ampicillin treated CTXM-15 positive strains.

In the current study, the expression of CTXM-15 gene was analysed in all the six E. coli strains. The E. coli CTXM-15 positive strains were treated with CcAgNps and strains without treatment were used as control. The genomic DNA was isolated from both the control and treated strains using heat lysis method. The DNA was amplified with CTX-M-15 gene primers for both the control and $\mathrm{CcAgNp}$ treated strains. The expression of CTXM-15 was down regulated in $\mathrm{CcAgNps}$ treated strains when compared

A MIC

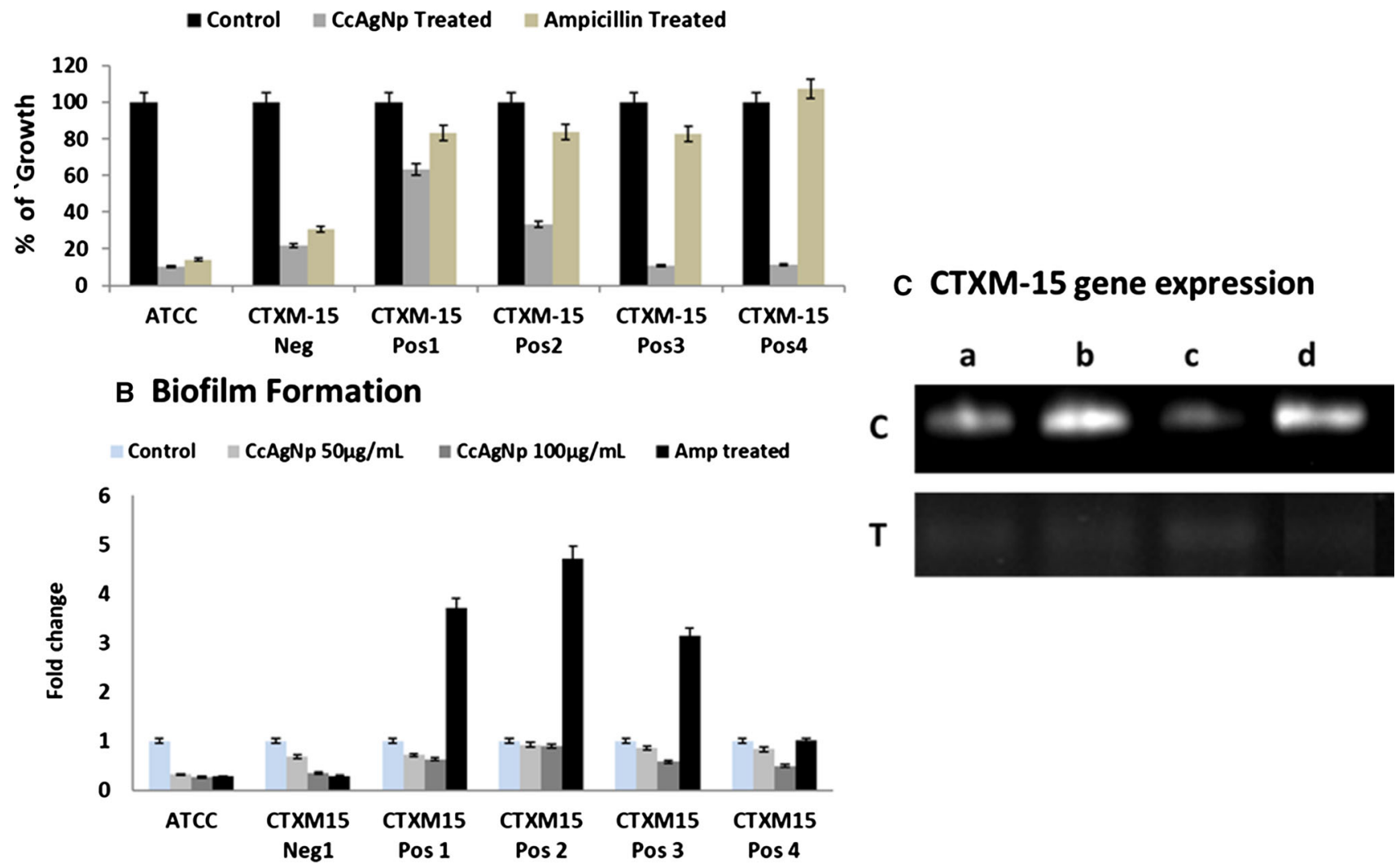

Fig. 2 A Percentage of bacterial growth of $\mathrm{CcAgNp}$ treated strains when compared to control and ampicillin treated strains. B Fold change indicating the inhibition of biofilm formation in CcAgNps treated strains when compared to control and ampicillin treated.

C Expression of CTXM-15 gene analysis: $\mathbf{C}$ indicates the control of four CTXM-15 positive strains a, b, c, d, T indicates the CcAgNp treated strains, which shows the down regulation of CTXM-15 gene expression in $\mathrm{a}, \mathrm{b}, \mathrm{c}, \mathrm{d}$ strains, when compare with the control strains 
to the control strains (Fig. 2C). The results suggested that CcAgNps can control the CTXM-15 gene expression in CTXM-15 positive $E$. coli strains. The silver nanoparticles from $C$. citratus extract constitute several antimicrobial phytochemical components which may play major role in inhibiting the expression of CTXM-15 gene expressing E. coli $[16,25,26]$.

\section{Conclusion}

The colloidal CcAgNps were synthesized, characterized and tested for its antibacterial and antibiofilm activity. CcAgNps showed enhanced antimicrobial activity against CTXM-15 expressing E. coli. The biofilm formation in CTXM-15 positive strains has been reduced when compared to control. CcAgNP treatment downregulated the expression of CTXM15 gene suggests that $\mathrm{CcAgNps}$ are regulating the virulence of $E$. coli at molecular level. The present work provides significance in the development of $\mathrm{CcAgNp}$ nanoparticles which can combat against the CTXM-15 expressing microbes. At the global level, development of antimicrobial resistant strains leads towards the search for novel alternatives to antibiotics, to break the antibiotic resistance in pathogenic microbial strains, which should be cost effective, biodegradable, ecofriendly. Hence, CcAgNps have the potential to control the growth and virulence factors in CTXM-15 positive human pathogenic E. coli.

Acknowledgements Ranjani S. is thankful to B.S. Abdur Rahman Institute of Science \& Technology, Chennai for providing research facilities in school of life sciences. The authors gratefully acknowledge Department of Science and Technology (GoI), New Delhi supported National Facility for Drug Development for Academia, Pharmaceutical and Allied Industries (NFDD) (Ref No. VI- D\&P/349/ 10-11/TDT/1 Dt: 21.10 .2010$)$. The authors also gratefully acknowledge the Ministry of Science and Technology, Department of Science and Technology (KIRAN Division) (GoI), New Delhi. (Ref No. DST/ WOS-B/2018/1583-HFN (G)).

\section{Compliance with Ethical Standards}

Conflict of interest Authors have no conflict of interest.

\section{References}

1. J. Behboud, E. Amirreza, M. A. Babak, and H. Zarifeh (2012). American-Eurasian J. Agric. Environ. Sci. 12, (8), 1042-1046. https://doi.org/10.5829/idosi.aejaes.2012.12.08.6551.

2. Sukriti Dutta, Sunita Munda, Mohan Lal, and P. R. Bhattacharyya (2016). Indian J. Sci. Technol. 9, 46. https://doi.org/10.17485/ijst/ 2016/v9i46/87046.

3. T. Mosquera, P. Noriega, J. Cornejo, and M. L. Pardo (2016). Int. J. Phytocosmet. Nat. Ingredients. 3, 7. https://doi.org/10.15171/ ijpni.2016.07.
4. S. M. Sousa, P. S. Silva, and L. F. Viccini (2010). Anias da Academia Brasileira de Ciencias. 82, (2), 305-331. https://doi. org/10.1590/S0001-37652010000200006.

5. M. I. Naik, B. A. Fomda, E. Jaykumar, and J. A. Bhat (2010). Asian Pac. J. Trop. Med. 3, 535-538. https://doi.org/10.1016/ S1995-7645(10)60129-0.

6. K. Anandalakshmi, J. Venugobal, and V. Ramasamy (2016). Appl. Nanosci. 6, 399-408. https://doi.org/10.1007/s13204-0150449-z.

7. S. A. Masurkar, P. R. Chaudhari, V. B. Shidore, and S. P. Kamble (2011). Micro Nano Lett. 3, 189-194. https://doi.org/10.1007/ BF03353671.

8. T. C. Prathna, N. Chandrasekaran, A. M. Raichur, and A. Mukherjee (2011). Colloid Surf. B. 82, 152-159. https://doi.org/ 10.1016/j.colsurfb.2010.08.036.

9. X. F. Zhang, Z. G. Liu, W. Shen, and S. Gurunathan (2016). Int. J. Mol. Sci. 17, 1534.

10. K. S. Siddiqi, A. Husen, and R. Rao (2018). J Nanobiotechnol. 16, 14. https://doi.org/10.1186/s12951-018-0334-5.

11. B. Das, S. K. Dash, D. Mandal, J. Adhikary, S. Chattopadhyay, S. Tripathy, A. Dey, S. Manna, S. K. Dey, D. Das, and S. Roy (2016). BLDE Univ. J. Health Sci. 1, 89.

12. H. Shameli, M. B. Ahmad, and A. Zamanian (2012). Int. J. Nanomedicine. 7, 5603-5610.

13. T. Akther, M. S. Khan, and H. Srinivasan (2018). Nano Biomed. Eng. 10, 250-257.

14. C. Chiluisa-Guacho, J. Escobar-Perez, and M. Dutra-Asensi (2018). Pathogens. 2, 42. https://doi.org/10.3390/ pathogens7020042.

15. S. Shaikh, J. Fatima, S. Shakil, S. M. Rizvi, and M. A. Kamal (2014). Saudi J. Biol. Sci. 22, (1), 90-101. https://doi.org/10. 1016/j.sjbs.2014.08.002.

16. R. Ubaid, S. Priya, A. Parveen, S. K. Sah, and S. Hemalatha (2018). BMC Complement Altern Med 18, 244.

17. G. Peirano, D. Richardson, and J. Nigrin (2010). Antimicrob. Agents Chemother. 54, (3), 1327-1330.

18. A. Tahira, S. Priya, S. Sarojkumar, M.S. Khan, and S. Hemalatha (2019). J. Nanostruct. 9, (2), 376-383. https://doi.org/10.22052/ JNS.2019.02.019.

19. S. Kaviya, J. Santhanalakshmi, B. Viswanathan, J. Muthumary, and K. Srinivasan (2011). Spectrochem. Acta A Mol. Biomol. Spectrosc. 79, 594-598.

20. A. A. Dashti, M. M. Jadaon, M. A. Abdulsamad, and H. M. Dashti (2009). Kuwait Med. J. 41, 117-122.

21. S. K. Sah, U. Rasool, and S. Hemalatha (2018). Asian J. Pharm. Clin. Res. 11, (7), 368-374. https://doi.org/10.22159/ajpcr.2018. v11i7.25664.

22. F. K. Alsammarraie, W. Wang, P. Zhou, A. Mustapha, and M. Lin (2018). Colloid Surf. B 171, 398-405.

23. Soheyla Honary and F. Zahir (2013). Trop. J. Pharm. Res. 12, 2. https://doi.org/10.4314/tjpr.v12i2.20.

24. K. Ali, B. Ahmed, S. Dwivedi, Q. Saquib, A. A. Al-Khedhairy, and J. Musarrat (2015). PLoS ONE. 10, 7.

25. L. N. Andrade, T. E. S. Siqueira, R. Martinez, and A. L. C. Darini (2018). Front Microbiol. 9, 539. https://doi.org/10.3389/fmicb. 2018.00539.

26. T. Akther and S. Hemalatha (2019). Bionanoscience. https://doi. org/10.1007/s12668-019-0607-y (in Press).

Publisher's Note Springer Nature remains neutral with regard to jurisdictional claims in published maps and institutional affiliations. 